\title{
Fourier Decompositions of Graphs with Symmetries and Equitable Partitions
}

Darren Scott Lund

Brigham Young University

Follow this and additional works at: https://scholarsarchive.byu.edu/etd

Part of the Physical Sciences and Mathematics Commons

\section{BYU ScholarsArchive Citation}

Lund, Darren Scott, "Fourier Decompositions of Graphs with Symmetries and Equitable Partitions" (2021). Theses and Dissertations. 8925.

https://scholarsarchive.byu.edu/etd/8925

This Thesis is brought to you for free and open access by BYU ScholarsArchive. It has been accepted for inclusion in Theses and Dissertations by an authorized administrator of BYU ScholarsArchive. For more information, please contact ellen_amatangelo@byu.edu. 
Fourier Decompositions of Graphs with Symmetries and Equitable Partitions

Darren Scott Lund

A thesis submitted to the faculty of Brigham Young University

in partial fulfillment of the requirements for the degree of

Master of Science

Benjamin Webb, Chair

Emily Evans

Mark Kempton

Department of Mathematics

Brigham Young University

Copyright (c) 2021 Darren Scott Lund

All Rights Reserved 


\begin{abstract}
Fourier Decompositions of Graphs with Symmetries and Equitable Partitions

Darren Scott Lund

Department of Mathematics, BYU

Master of Science

We show that equitable partitions, which are generalizations of graph symmetries, and Fourier transforms are fundamentally related. For a partition of a graph's vertices we define a Fourier similarity transform of the graph's adjacency matrix built from the matrices used to carryout discrete Fourier transformations. We show that the matrix (graph) decomposes into a number of smaller matrices (graphs) under this transformation if and only if the partition is an equitable partition. To extend this result to directed graphs we define two new types of equitable partitions, equitable receiving and equitable transmitting partitions, and show that if a partition of a directed graph is both, then the graph's adjacency matrix will similarly decomposes under this transformation. Since the transformation we use is a similarity transform the collective eigenvalues of the resulting matrices (graphs) is the same as the eigenvalues of the original untransformed matrix (graph).
\end{abstract}

Keywords: Discrete Fourier Transform, graph, network, Fourier Similarity Transform, equitable partition, graph similarity 


\section{ACKNOWLEDGEMENTS}

In addition to Dr. Webb, special thanks to Joseph Drapeau and Reilly Bex for working through the details on this with me. 


\section{Contents}

Contents

List of Figures

1 Introduction 1

2 Equitable Partitions $\quad 4$

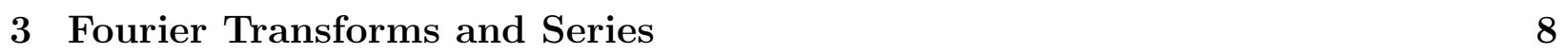

4 Proof of the Graph Decomposition Theorem 17

5 Generalization of Equitable Decompositions to Directed Graphs and Consequences

6 Fourier Decompositions over Symmetries $\quad 32$

$\begin{array}{lll}7 & \text { Conclusion } & 35\end{array}$

$\begin{array}{ll}\text { Bibliography } & 37\end{array}$ 


\section{LiST OF FiguRES}

2.1 An example of an equitable partition and the corresponding adjacency matrix. 6

3.1 An example of the continuous Fourier decomposition. . . . . . . . . . . . 9

3.2 The Fourier Graph Decomposition of Figure 2.1 . . . . . . . . . . . . . . . . 14

3.3 The Paul Revere Network . . . . . . . . . . . . . . . . . 16

3.4 The FST of the Paul Revere Network . . . . . . . . . . . . . . . . . 17

5.1 A directed graph that is both an ERP and an ETP but whose undirected

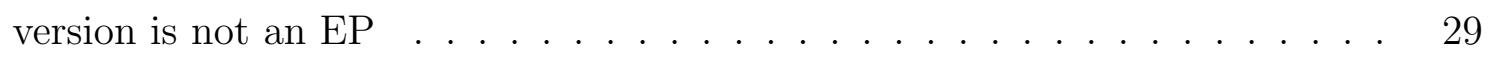

6.1 The FST of Figure 2.1 but with a different partition $\pi$. . . . . . . . . . . 33 


\section{Chapter 1. Introduction}

Graphs are used to represent many types of systems. This includes most networks studied in the social, technological, and natural sciences $[6,15,21,26]$. It also includes the data structures used in computer science [5, 12], the structure of interaction within machine learning algorithms $[7,16,18,20]$, and the physical structure of molecules in chemistry $[1,4]$ to name a few.

The goal of spectral graph theory is to understand how this structure influences the graph's spectrum, i.e. the eigenvalues of an associated matrix, and conversely what these eigenvalues say about the structure of the graph and the corresponding system. The particular type of structure we consider in this paper are graph symmetries or more generally the equitable partitions of a graph. In a simple graph $G=(V, E)$, which is an undirected unweighted graph without self-loops, an equitable partition $\pi=\left\{V_{1}, V_{2}, \ldots, V_{k}\right\}$ of the graph is a partition of the graph's vertices $V$ such that any vertex in $V_{i}$ is a neighbor to the same number of vertices in $V_{j}$ irregardless of which vertex we choose.

Originally, interest in equitable partitions was due to the spectral properties preserved by these partitions $[9,13]$. More recently equitable partitions have been shown to be ubiquitous in real-networks [19]. These partitions are also known to impact the dynamical processes that take place in such systems, including the formation of synchronizing clusters in dynamical network models $[22,25]$ etc. Additionally, and most related to the results of this paper, the equitable partitions induced by graph symmetries have been used to decompose graphs into a collection of smaller graphs while preserving the graph's spectrum $[2,10,11]$.

In what is typically considered a very separate part of mathematics, Fourier transforms are used to take a periodic function or signal $f:[0, T] \rightarrow \mathbb{R}$ and decompose this function into a sum of simple waves. In practice, the signal is first sampled then transformed via a discrete Fourier transform (DFT). Since Fourier transforms are linear this amounts to a matrix transformation of the discretized signal. Such transformations can be done very 
efficiently and are useful in numerous applications including image processing, audio filtering, magnetic resonance imaging, and many more [3, 17, 23, 28].

The main result of this paper is that equitable partitions and Fourier transformations are fundamentally related. Specifically, given a partition $\pi$ of an undirected graph's vertices we define a Fourier similarity transform $F_{\pi}(A)=W_{\pi}^{-1} A W_{\pi}$ (see Definition 3.1) of the graph's adjacency matrix $A$, where the matrix $W_{\pi}$ is built from the matrices used to carry out DFTs. If the partition $\pi$ is an equitable partition then some permuted version of the matrix decomposes into the direct sum of matrices

$$
P^{-1} F S T_{\pi}(A) P=A_{\pi} \oplus B
$$

where $P$ is a known permutation matrix (see Lemma 3.3), $A_{\pi}$ is the divisor matrix associated with the equitable partition (see Definition 2.2), and $B$ is some other matrix. In fact, the converse of this also holds so that a Fourier similarity transform results in the decomposition $A_{\pi} \oplus B$ if and only if $\pi$ is an equitable partition (see Theorem 3.2). That is, similar to the way a Fourier transform decomposes a periodic function into the sum of other periodic functions this transform decomposes matrices (graphs) that have an equitable partition into the direct sum of other smaller matrices (graphs).

If a graph is directed the situation is more complicated and we need to consider two generalizations of an equitable partition. The first is an equitable receiving partition (ERP), which is the same as an equitable partition except we only care about incoming edges (see Definition 5.2). The second is an equitable transmitting partition (ETP), for which we only care about outgoing edges (see Definition 5.3). If a directed graph has an ERP $\pi$ then its adjacency matrix no longer strictly decomposes into the direct sum of matrices but takes the upper block-triangular form

$$
P^{-1} F S T_{\pi}(A) P=\left[\begin{array}{cc}
A_{\pi} & C \\
0 & B
\end{array}\right]
$$


If a directed graph has an ETP $\pi$ then its adjacency matrix has the lower block-triangular form

$$
P^{-1} F S T_{\pi}(A) P=\left[\begin{array}{cc}
A_{\pi} & 0 \\
C & B
\end{array}\right] .
$$

If a directed graph has a partition $\pi$ that is both an ERP and an ETP it follows that $P^{-1} F S T_{\pi}(A) P=A_{\pi} \oplus B$. That is, in this case we have a strict decomposition of the graph's adjacency matrix. Moreover, like Theorem 3.2 the converse to this result also holds (see Theorem 5.4). Each of these decompositions can be done on any graph with an adjacency $\operatorname{matrix} A \in \mathbb{C}^{n \times n}$.

The structure of the graph's equitable partition is given by the divisor matrix $A_{\pi}$. Using the decompositions given in Theorems 3.2 and 5.4 one can obtain the remaining part of the graph given by the matrix $B$ and analyze its properties. Since these decompostions are based on similarity transforms these transforms preserve the spectrum of the original graph (matrix), i.e. are isospectral transformations of the original graph (matrix). Specifically, for any equitable partition, ERT, or ETP the eigenvalues $\sigma(A)=\sigma\left(A_{\pi}\right) \cup \sigma(B)$ (see Corollary 3.6 and Corollary 5.7). Thus, the Fourier decompositions introduced in this paper are potential tools for analyzing both the spectral and structural properties of the original graph (matrix).

It is worth mentioning that a special case of an equitable partition is a graph symmetry. The papers $[2,10,11]$ describe a way of decomposing a graph $G$ with respect to any one of its symmetries. This method of decomposition is referred to as an equitable decomposition as the resulting transformation similarly contains the divisor matrix $A_{\pi}$. One limitation of this previous theory is that a graph symmetry is required. Since not every equitable partition comes from a symmetry (see Example 3.5) it is not possible to equitably decompose a graph with respect to this type of equitable partition, which the theory presented in this paper allows us to do.

We note that the Fourier decompositions we present can be done over any equitable partition and even more generally over the new class(es) of partitions, ERPs and ETPs, 
introduced here. Moreover, these decompositions are computationally quite simple when compared to equitable decompositions as they require only a single similarity transform as opposed to the multiple similarlity transforms required, in general, for an equitable decomposition (for more details see $[2,10,11]$.

In Chapter 2 we describe the basic ideas involving equitable partitions. In Chapter 3 we introduce the notion of a Fourier similarity transform and describe how this transform can be used to decompose an undirected graph (symmetric matrix) if and only if it has an equitable partition. In the following chapter we prove this result. In Chapter 5 we generalize the results of Chapter 3 to directed graphs (general matrices) by introducing the concepts of equitable receiving and transmitting partitions. In Chapter 6 we discuss the special case of equitable partitions derived from graph symmetries and connections to the theory of equitable decompositions. Chapter 7 concludes with a few closing remarks including open questions regarding Fourier decompositions.

\section{Chapter 2. Equitable Partitions}

Graphs are used to represent many types of systems. The key idea that a graph captures is the structure of relationships or interactions between a collection of objects. The relationships could be social relationships between individuals, the physical connections between routers in the Internet, the synapses that couple neurons in the brain, or the interdependence of variables given by a function $F: \mathbb{R}^{n} \rightarrow \mathbb{R}^{n}$. A graph $G=(V, E, \omega)$ is composed of a vertex set $V=\{1,2, \ldots, n\}$, an edge set $E$, and a function $\omega: E \rightarrow \mathbb{C}$ used to weight the edges $E$ of the graph. The vertex set $V$ represents the system's collection of objects and the edges $E$ the links or interactions between these objects. The function $\omega$ can be though of as measuring the strength of these interactions. For instance, if the graph represents a social network the weights might be the frequency of interaction between individuals.

In some systems there is a sense of direction to the system's set of interactions. For example, in a citation network, citing a paper is not the same as being cited by a paper. 
In the graph representing a citation network there is a directed edge from the paper to the paper it is citing. In this case the graph is a directed graph as every edge has a clearly defined direction. If the edges of the graph have no specified direction, i.e. are undirected, then the graph is referred to as an undirected graph. We note that an undirected graph can be considered to be a directed graph by replacing each of its undirected edges by two directed edges: one that points from the first to the second vertex and the other that points from the second back to the first. Hence, any graph can be considered to be a directed graph.

Using this coinvention, let $G=(V, E, \omega)$ be a graph on $|V|=n$ vertices. Its adjacency matrix, $A=A(G) \in \mathbb{C}^{n \times n}$ has entries given by

$$
A_{i j}= \begin{cases}\omega\left(e_{i j}\right) \neq 0 & \text { if } e_{i j} \in E \\ 0 & \text { otherwise }\end{cases}
$$

where $e_{i j}$ is the directed edge from vertex $i$ to vertex $j$. Here an edge $e_{i i} \in E$ is a loop of the graph. The eigenvalues of the graph $G$ are the eigenvalues $\sigma(A)$ of its adjacency matrix. A graph is undirected if and only if its adjacency matrix is symmetric.

Since $\omega\left(e_{i j}\right) \in \mathbb{C}$ is the weight of the edge $e_{i j}$ the matrix $A=A(G)$ is, technically speaking, the weighted adjacency matrix of a graph $G=(V, E, \omega)$. For simplicity we will refer to $A$ as the graph's adjacency matrix. The reason for this is that an unweighted graph $G=(V, E)$ can be considered to be a special case of a weighted graph where the unweighted edges of the graph are given unit weight. Without loss in generality then, Equation (2.1) defines the adjacency matrix for any graph whether it is directed or undirected, weighted or unweighted.

Despite the generality in which we have defined a graph a large fraction of real-world systems are represented by simple graphs, which are unweighted, undirected graphs without loops. This includes, for example, the large majority of networks studied in the social, technological, and natural sciences $[6,21,26]$. The adjacency matrix of a simple graph is a symmetric, zero-one matrix, that has an all-zero diagonal. For example, the graph 


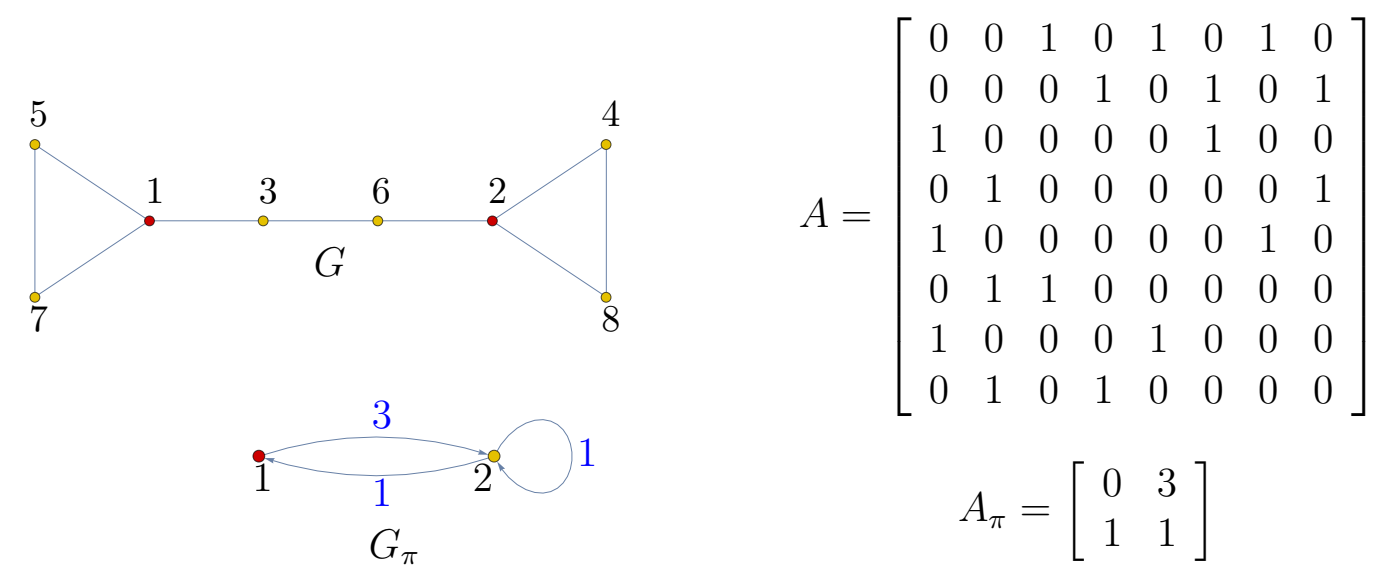

Figure 2.1: Top Left: A simple graph $G=(V, E)$ with equitable partition $\pi=\left\{V_{1}, V_{2}\right\}$ where $V_{1}=\{1,2\}$ and $V_{2}=\{3,4,5,6,7,8\}$ are shown in red and yellow, respectively. Top Right: The adjacency matrix $A=A(G)$ is shown. Bottom Left: The divisor graph $G_{\pi}$ is shown with vertices 1 (red) and 2 (yellow) corresponding to the elements $V_{1}$ and $V_{2}$, respectively, of the equitable partition $\pi$. Edge weights are shown in blue. Bottom Right: The divisor matrix $A_{\pi}$ of $A$.

$G=(V, E)$ in Figure 2.1 (top left) is a simple graph with the zero-one adjacency matrix $A \in\{0,1\}^{n \times n}$ shown in Figure 2.1 (top right).

In such real-world systems structure and function are thought to be intrinsically related. Structural motifs, i.e. statistically significant subgraphs, community structure, graph components of a system all determine how quantities such as traffic, information, and contagions move through the system or how effort is coordinated [5, 21, 24]. The particular type of structure we consider in this paper is an equitable partition. Before defining an equitable partition, it is convenient to define a specific value related to the graph.

Definition 2.1. For an undirected graph $G=(V, E, \omega)$ with a vertex partition $\pi=$ $\left\{V_{1}, V_{2}, \ldots, V_{k}\right\}$ (not necessarily an equitable partition), define

$$
D_{a b}=\frac{1}{\left|V_{a}\right|} \sum_{i \in V_{a}}\left(\sum_{j \in V_{b}} A_{i j}\right)
$$

$D_{a b}$ can be thought of as the smallest number of connections from a node in partition $V_{a}$ to a node in partition $V_{b}$.

We are now ready to define an equitable partition as follows. 
Definition 2.2. (Equitable Partition) An equitable partition of an undirected graph $G=(V, E, \omega)$ is a partition $\pi=\left\{V_{1}, V_{2}, \ldots, V_{k}\right\}$ of the vertices $V$ with the property that for all $a, b \in\{1,2, \ldots, k\}$ the sum

$$
\sum_{j \in V_{b}} A_{i j}=(D)_{a b}
$$

is constant for any $i \in V_{a}$. The matrix $D=A_{\pi} \in \mathbb{R}^{k \times k}$ is called the divisor matrix of $A$ associated with $\pi$. The graph $G_{\pi}$ with adjacency matrix $A_{\pi}$ is the divisor graph of $G$.

In the case of a simple graph $G=(V, E)$ an equitable partition is a partition $\pi=$ $\left\{V_{1}, V_{2}, \ldots, V_{k}\right\}$ where the following is true. Any vertex $i \in V_{a}$ is adjacent to the same number of vertices in $V_{b}$ for all $a, b \in\{1,2, \ldots, k\}$ irrespective of which vertex $i$ we choose from $V_{a}$. The entry $\left(A_{\pi}\right)_{a b}=D_{a b}$ in the divisor matrix is simply the number of vertices any vertex in $V_{a}$ is adjacent to in $V_{b}$.

An intuitive understanding of what Definition 2.2 says is that for any two rows of the adjacency matrix $A$ corresponding to vertices in the same partition element $V_{a}$, then the partial row sums taken by adding the elements in the columns corresponding to vertices of partition element $V_{b}$ are all equal.

Example 2.3. Consider the graph $G$ in Figure 2.1 (left). The graph has the equitable partition $\pi=\left\{V_{1}, V_{2}\right\}$ consisting of the two elements $V_{1}=\{1,2\}$ and $V_{2}=\{3,4,5,6,7,8\}$ shown in red and yellow, respectively. The partition $\pi$ is an equitable partition of $G$ since every vertex in $V_{1}$ is adjacent to zero vertices in $V_{1}$ and three vertices in $V_{2}$, while every vertex in $V_{2}$ is adjacent to one vertex in $V_{1}$ and one vertex in $V_{2}$. As a consequence, the divisor matrix is

$$
A_{\pi}=\left[\begin{array}{ll}
0 & 3 \\
1 & 1
\end{array}\right] .
$$

The divisor graph $G_{\pi}$ and associated divisor matrix $A_{\pi}$ are shown in Figure 2.1 (bottom left and right, respectively).

Equitable partitions have received considerable attention in the literature. Originally this 
interest was due to the spectral properties preserved by these partitions. Likely the most well-known of these is that if $A_{\pi}$ is a divisor matrix of $A$ then $\sigma\left(A_{\pi}\right) \subseteq \sigma(A)[9,13]$. More recently equitable partitions have been shown to be a hallmark of real-world networks [19]. Equitable partitions can also have an impact on the function and processes that can take place on these networks. This includes the formation of synchronizing clusters in dynamical network models [22, 27, 25]. Additionally, as discussed in Chapter 6, equitable partitions have been used to decompose graphs with symmetries [2, 10, 11].

In the following chapter we describe how Fourier transformations can be used to decompose a graph into a number of smaller graphs if the graph has an equitable partition.

\section{Chapter 3. Fourier Transforms and Series}

The standard use of a Fourier transform is to take a periodic function $f:[0, T] \rightarrow \mathbb{R}$ with period $T$ and decompose it into a sum of simple waves given by sines and cosines

or more generally complex exponentials [14]. In particular, it is possible to transform a square-integrable function $f \in L^{2}([0, T], \mathbb{R})$ into its Fourier series

$$
f(t)=\sum_{k=-\infty}^{\infty} c_{k} e^{2 \pi i\left(\frac{k}{T}\right) t}
$$

where

$$
c_{k}=\frac{1}{T} \int_{0}^{T} f(t) e^{-2 \pi i\left(\frac{k}{T}\right) t} d t
$$

For instance, the function $f(t)$ shown in Figure 3.1(a) can be decomposed into the sum

$$
f(t)=\frac{7}{2} i e^{-10 i t}+\frac{5}{2} i e^{-4 i t}+i e^{-3 i t}-i e^{3 i t}-\frac{5}{2} i e^{4 i t}-\frac{7}{2} i e^{10 i t}
$$

using (3.1) and (3.2). In terms of real real-valued functions $f(t)$ has the Fourier decomposition $f(t)=2 \sin (3 t)+5 \sin (4 t)+7 \sin (10 t)$, which is shown in Figures 3.1(b)-(d).

In practice the function $f$ represents a continuous-time signal. The advantage to writing 


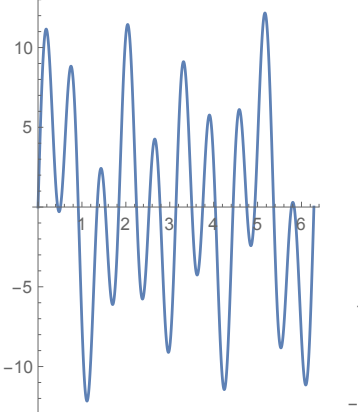

(a) $f(t)$

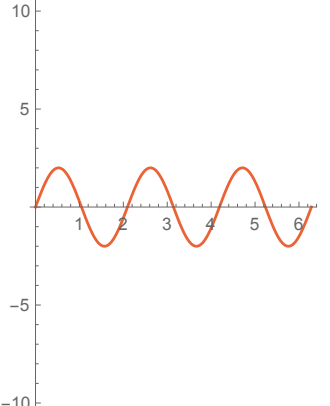

(b) $\hat{f}_{1}(t)$

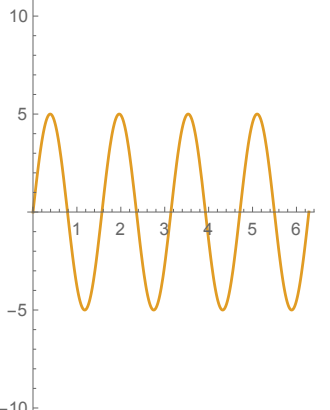

(c) $\hat{f}_{2}(t)$

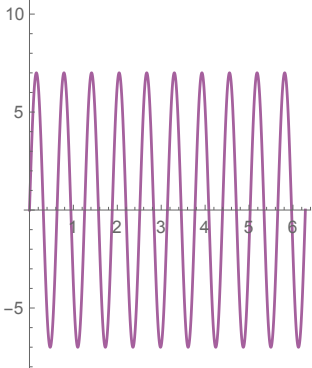

(d) $\hat{f}_{3}(t)$

Figure 3.1: The Fourier decomposition of the continuous time signal $f(t)$ into the sum $f(t)=\sum_{i=1}^{3} \hat{f}_{i}(t)$ where $\hat{f}_{1}(t)=2 \sin (3 t), \hat{f}_{2}(t)=5 \sin (4 t)$, and $\hat{f}_{3}(t)=7 \sin (10 t)$.

$f$ as in Equation (3.1) is that this gives us an effective way to analyze, filter, and compress the original signal. In any technological application signals are discrete rather than continuous. In order to apply Fourier methods to these continuous signals, these signals are converted to discrete signals via sampling. A sample of a continuous function $f$ is an $n$-tuple

$$
\mathbf{x}=\left(f\left(t_{1}\right), f\left(t_{2}\right), \ldots, f\left(t_{n}\right)\right) \in \mathbb{R}^{n}
$$

where $0=t_{1}<t_{2}<\cdots<t_{n}<t_{n+1}=T$.

For a discrete-time signal $\mathbf{x} \in \mathbb{R}^{n}$ the analogue to the Fourier transform is the discrete Fourier transform (DFT). The DFT is a linear transformation from $\mathbb{R}^{n}$ to $\mathbb{R}^{n}$, or more generally from $\mathbb{C}^{n}$ to $\mathbb{C}^{n}$, and can therefore be expressed in terms of a matrix $W_{n}$. This discrete Fourier transform of the signal $\mathbf{x}$ is given by $\operatorname{DFT}(\mathbf{x})=W_{n} \mathbf{x}$ where

$$
W_{n}=\left[\begin{array}{cccc}
\omega_{n}^{0} & \omega_{n}^{0} & \ldots & \omega_{n}^{0} \\
\omega_{n}^{0} & \omega_{n}^{1} & \ldots & \omega_{n}^{n-1} \\
\omega_{n}^{0} & \omega_{n}^{2} & \ldots & \omega_{n}^{2(n-1)} \\
\vdots & \vdots & \ddots & \vdots \\
\omega_{n}^{0} & \omega_{n}^{(n-1)} & \ldots & \omega_{n}^{(n-1)^{2}}
\end{array}\right] \in \mathbb{C}^{n \times n}
$$


in which $\omega_{n}$ is the $n$th primitive root of unity $\omega_{n}=e^{2 \pi i / n}$. The inverse of this matrix is given by $W_{n}^{-1}=n W_{n}^{H}=n \bar{W}$ where $W^{H}$ is the conjugate transpose of $W$ and $\bar{W}$ is its conjugate.

Because DFTs can be done efficiently using fast Fourier transforms (FFTs) they are useful in numerous applications including image processing, audio filtering, magnetic resonance imaging, and many more [3, 17, 23, 28]. To the best of the authors' knowledge DFTs have not, however, been used to decompose graphs or matrices. This is potentially because graphs and matrices are static objects, at least as they are typically defined and there is no obvious analogy between a sampled signal $\mathbf{x} \in \mathbb{R}^{n}$ and a graph $G$ with adjacency matrix $A \in \mathbb{R}^{n \times n}$.

In what follows, if a graph $G=(V, E, \omega)$ has an equitable partition $\pi=\left\{V_{1}, V_{2}, \ldots, V_{k}\right\}$ then, without loss in generality, we assume that the vertices of $G$ are numbered such that if $i \in V_{a}$ and $j \in V_{b}$ where $a<b$ then $i<j$. This amounts to relabelling the vertices of $G$ so that vertices in the same element of $\pi$ are labeled consecutively and the vertices in $V_{a}$ precede those in $V_{a+1}$ for $a=1,2, \ldots, k-1$. For example, the graph $G$ in Figure 2.1 has the equitable partition $\pi=\left\{V_{1}, V_{2}\right\}$ that respects this labeling as $V_{1}=\{1,2\}$ and $V_{2}=\{3,4,5,6,7,8\}$. This results in the adjacency matrix

$$
A=\left[\begin{array}{ll|llllll}
0 & 0 & 1 & 0 & 1 & 0 & 1 & 0 \\
0 & 0 & 0 & 1 & 0 & 1 & 0 & 1 \\
\hline 1 & 0 & 0 & 0 & 0 & 1 & 0 & 0 \\
0 & 1 & 0 & 0 & 0 & 0 & 0 & 1 \\
1 & 0 & 0 & 0 & 0 & 0 & 1 & 0 \\
0 & 1 & 1 & 0 & 0 & 0 & 0 & 0 \\
1 & 0 & 0 & 0 & 1 & 0 & 0 & 0 \\
0 & 1 & 0 & 1 & 0 & 0 & 0 & 0
\end{array}\right]=\left[\begin{array}{ll}
A[1,1] & A[1,2] \\
A[2,1] & A[2,2]
\end{array}\right]
$$

where $A[i, j]$ is the sub-matrix of $A$ with rows indexed by $V_{i}$ and columns indexed by $V_{j}$.

For a graph $G$ with equitable partition $\pi$ Definition 2.2 implies that the row sums of $A[i, j]$ are $\left[A_{\pi}\right]_{i j}=D_{i j}$ for every row. If $G$ is undirected then we also have that the column 
sums of $A[i, j]$ are $\left[A_{\pi}\right]_{j i}=D_{j i}$ for every column. This observation will be important in using Fourier transforms to decompose undirected graphs, i.e. graph whose adjacency matrix is symmetric (see Theorem 3.2).

Definition 3.1. (Fourier Similarity Transform) Let $G=(V, E, \omega)$ be an graph with vertex partition $\pi=\left\{V_{1}, V_{2}, \ldots, V_{k}\right\}$ in which $\left|V_{i}\right|=n_{i}$. Then the Fourier similarity transform (FST) of $A=A(G)$ is the transformation

$$
F S T_{\pi}(A)=W_{\pi}^{-1} A W_{\pi}
$$

where $W_{\pi}$ is the block-diagonal matrix

$$
W_{\pi}=W_{n_{1}} \oplus W_{n_{2}} \oplus \cdots \oplus W_{n_{k}}=\left[\begin{array}{cccc}
W_{n_{1}} & 0 & \ldots & 0 \\
0 & W_{n_{2}} & \ldots & 0 \\
\vdots & \vdots & \ddots & \vdots \\
0 & 0 & \ldots & W_{n_{k}}
\end{array}\right]
$$

The Fourier similarity transform of the graph $G$ is the graph $F S T_{\pi}(G)$ with adjacency matrix $F S T_{\pi}(A)$.

Note that even if $G$ is a simple graph then $F S T_{\pi}(G)$ will typically not be simple graph but a weighted directed graph (see Example 3.5).

The fundamental connection between equitable partitions and the discrete Fourier transform is stated in the following theorem.

Theorem 3.2. (Fourier Decompositions of Undirected Graphs) Let $G=(V, E, \omega)$ be an undirected graph with vertex partition $\pi=\left\{V_{1}, V_{2}, \ldots, V_{k}\right\}$. We can construct a permutation matrix $P$ such that the permuted Fourier similarity transform of $A=A(G)$ decomposes into the direct product

$$
P^{-1} F S T_{\pi}(A) P=A_{\pi} \oplus B \in \mathbb{C}^{n \times n}
$$

if and only if $\pi$ is an equitable partition of $G$. 
That is, a graph (matrix) can be decomposed into (the direct sum of) a number of smaller graphs (matrices) via a Fourier similarity transform only in the case that it has an equitable partition. The proof of Theorem 3.2 is given in the following chapter. To prove this theorem we use the following lemma, which describes how to construct the permutation matrix $P$.

Lemma 3.3. Let $G=(V, E, \omega)$ be an undirected graph with vertex partition $\pi=\left\{V_{1}, V_{2}, \ldots, V_{k}\right\}$. If $\left|V_{i}\right|=n_{i}$ then the matrix $P$ in Equation (3.5) is given by

$$
P=\left[\boldsymbol{e}_{1}, \boldsymbol{e}_{1+n_{1}}, \boldsymbol{e}_{1+n_{1}+n_{2}}, \ldots, \boldsymbol{e}_{1+\sum_{i=1}^{k-1} n_{i}}, \boldsymbol{e}_{2}, \boldsymbol{e}_{3}, \ldots, \boldsymbol{e}_{n_{1}}, \boldsymbol{e}_{n_{1}+2}, \ldots, \boldsymbol{e}_{n_{1}+n_{2}}, \boldsymbol{e}_{n_{1}+n_{2}+2}, \ldots, \boldsymbol{e}_{n}\right]
$$

where $\boldsymbol{e}_{i}$ is the $i^{\text {th }}$ column of $I_{n}$, the $n \times n$ identity matrix.

In other words, the first $k$ columns of $P$ are chosen as $\mathbf{e}_{1}, \mathbf{e}_{1+n_{1}}, \mathbf{e}_{1+n_{1}+n_{2}}, \ldots, \mathbf{e}_{1+\sum_{i=1}^{k-1} n_{i}}$ and the remaining columns are all the previously unused columns of the identity matrix in ascending order. Since the purpose of the permutation matrix is to separate $A_{\pi}$ from $B$, only the order of the first $k$ columns is important. The others are chosen sequentially for convenience, but do not need to be in any particular order for the results of this paper to hold.

Example 3.4. Suppose we have the partition sizes $\left\{n_{i}\right\}_{i=1}^{4}=\{2,1,2,1\}$, i.e. $n_{1}=2, n_{2}=1$, $n_{3}=2$, and $n_{4}=1$. Then

$$
P=\left[\begin{array}{llllll}
\mathbf{e}_{1} & \mathbf{e}_{3} & \mathbf{e}_{4} & \mathbf{e}_{6} & \mathbf{e}_{2} & \mathbf{e}_{5}
\end{array}\right]
$$

since $\mathbf{e}_{1+n_{1}}=\mathbf{e}_{1+2}=\mathbf{e}_{3}, \mathbf{e}_{1+n_{1}+n_{2}}=\mathbf{e}_{1+2+1}=\mathbf{e}_{4}$, and $\mathbf{e}_{1+n_{1}+n_{2}+n_{3}}=\mathbf{e}_{1+2+1+2}=\mathbf{e}_{6}$. Since $k=4$, following this we insert the unused columns of $I$ into $P$ in ascending order.

Remark. In order to fully understand why we use the matrix $P$, it is useful to see what exactly the transform $B=P^{T} A P$ does to a matrix $A$. Let $G=(V, E, \omega)$, and $\pi=\left\{V_{1}, V_{2}, \ldots, V_{k}\right\}$ 
be a partition of the vertices of the graph $A(G)$. We then write

$$
A=\left[\begin{array}{cccc}
A[1,1] & A[1,2] & \ldots & A[1, k] \\
A[2,1] & A[2,2] & \ldots & A[2, k] \\
\vdots & \vdots & \ddots & \vdots \\
A[k, 1] & A[k, 2] & \ldots & A[k, k]
\end{array}\right]
$$

as we have used before, so $A[a, b] \in \mathbb{C}^{n_{a} \times n_{b}}$ where $n_{i}=\left|V_{i}\right|$, and $n_{0}=1$ for convenience. Then if $1 \leq i, j \leq k$, and $p_{i}$ is the $i^{\text {th }}$ column of $P$, we can write

$$
[B]_{i j}=p_{i}^{T} A p_{j}=\mathbf{e}_{\sum_{s=0}^{T-1} n_{s}}^{T} A \mathbf{e}_{\sum_{s=0}^{j-1} n_{j}}=A[i, j]_{1,1}
$$

So $P$ takes the top left entry of $A[i, j]$ and makes it the $i, j$ entry of $B$. Similarly, if $1 \leq i \leq k$ but $k<j \leq n$, then

$$
[B]_{i j}=p_{i}^{T} A p_{j}=\mathbf{e}_{\sum_{s=0}^{i-1} n_{s}}^{T} A p_{j}=A[i, l]_{1, k}
$$

for some $l, k$ dependent on $j$, and if $1 \leq j \leq k$ but $k<i \leq n$, then

$$
[B]_{i j}=p_{i}^{T} A p_{j}=p_{i}^{T} A \mathbf{e}_{\sum_{s=0}^{j-1} n_{s}}=A[l, j]_{k, 1}
$$

for some $l, k$ dependent on $i$. In other words, if $B=P^{T} A P$, then the first $k$ columns of $B$ are the first columns of each submatirx $A[i, j]$, and the first $k$ rows of $B$ are the first columns of each submatrix $A[i, j]$.

A second construction of a matrix $P$ is given in the following example describing how the graph in Figure 2.1 is decomposed using this method. 

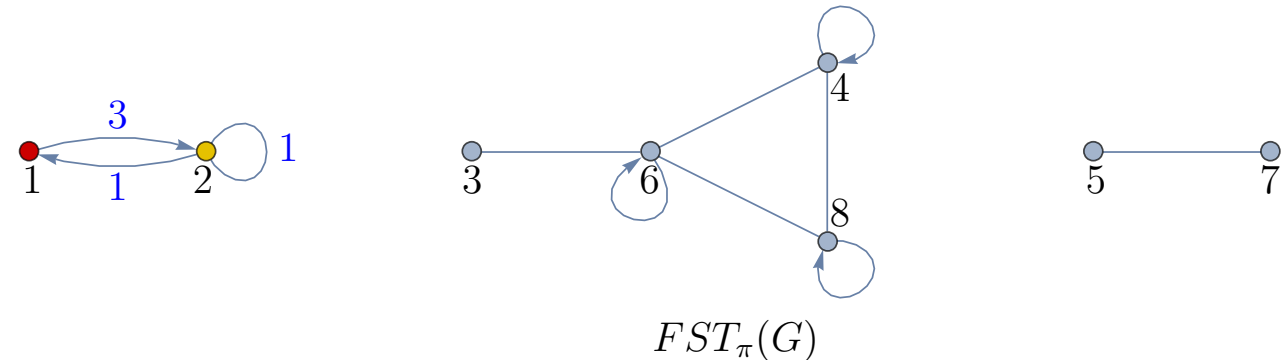

Figure 3.2: The Fourier Graph Decomposition $F S T_{\pi}(G)$ of the graph $G$ in Figure 2.1 over the equitable partition $\pi=\{\{1,2\},\{3,4,5,6,7,8\}\}$ is shown. The subgraph $G_{\pi}$ of the decomposition is shown (left) and is colored as in Figure 2.1. Edge weights of the graph are omitted except on the divisor graph $G_{\pi}$ and vertices are labeled so that the graph's adjacency matrix is given by (3.7).

Example 3.5. For the graph $G$ in Figure 2.1 with equitable partition $\pi=\{\{1,2\},\{3,4,5,6,7,8\}\}$ the matrix $W_{\pi}$ is given by

$$
W_{\pi}=\left[\begin{array}{cc}
W_{2} & 0 \\
0 & W_{6}
\end{array}\right]=\left[\begin{array}{cccccccc}
1 & 1 & 0 & 0 & 0 & 0 & 0 & 0 \\
1 & -1 & 0 & 0 & 0 & 0 & 0 & 0 \\
0 & 0 & 1 & 1 & 1 & 1 & 1 & 1 \\
0 & 0 & 1 & \frac{1}{2}+\frac{\sqrt{3}}{2} i & -\frac{1}{2}+\frac{\sqrt{3}}{2} i & -1 & -\frac{1}{2}-\frac{\sqrt{3}}{2} i & \frac{1}{2}-\frac{\sqrt{3}}{2} i \\
0 & 0 & 1 & -\frac{1}{2}+\frac{\sqrt{3}}{2} i & -\frac{1}{2}-\frac{\sqrt{3}}{2} i & 1 & -\frac{1}{2}+\frac{\sqrt{3}}{2} i & -\frac{1}{2}-\frac{\sqrt{3}}{2} i \\
0 & 0 & 1 & -1 & 1 & -1 & 1 & -1 \\
0 & 0 & 1 & -\frac{1}{2}-\frac{\sqrt{3}}{2} i & -\frac{1}{2}+\frac{\sqrt{3}}{2} i & 1 & -\frac{1}{2}-\frac{\sqrt{3}}{2} i & -\frac{1}{2}+\frac{\sqrt{3}}{2} i \\
0 & 0 & 1 & \frac{1}{2}-\frac{\sqrt{3}}{2} i & -\frac{1}{2}-\frac{\sqrt{3}}{2} i & -1 & -\frac{1}{2}+\frac{\sqrt{3}}{2} i & \frac{1}{2}+\frac{\sqrt{3}}{2} i
\end{array}\right]
$$

The Fourier similarity transform of the matrix $A=A(G)$ is then the matrix 


$$
F T_{\pi}(A)=\left[\begin{array}{cc|cccccc}
0 & 0 & 3 & 0 & 0 & 0 & 0 & 0 \\
0 & 0 & 0 & 0 & 0 & 3 & 0 & 0 \\
\hline 1 & 0 & 1 & 0 & 0 & 0 & 0 & 0 \\
0 & 0 & 0 & -\frac{2}{3} & 0 & -\frac{2}{3} & 0 & \frac{1}{3} \\
0 & 0 & 0 & 0 & 0 & 0 & 1 & 0 \\
0 & 1 & 0 & -\frac{2}{3} & 0 & \frac{1}{3} & 0 & -\frac{2}{3} \\
0 & 0 & 0 & 0 & 1 & 0 & 0 & 0 \\
0 & 0 & 0 & \frac{1}{3} & 0 & -\frac{2}{3} & 0 & -\frac{2}{3}
\end{array}\right]
$$

and the permutation matrix $P$ is

$$
P=\left[\begin{array}{llllllll}
\mathbf{e}_{1} & \mathbf{e}_{3} & \mathbf{e}_{2} & \mathbf{e}_{4} & \mathbf{e}_{5} & \mathbf{e}_{6} & \mathbf{e}_{7} & \mathbf{e}_{8}
\end{array}\right]
$$

which is found by using Lemma 3.3. The permuted similarity transform is

$$
P^{-1} F S T_{\pi}(A) P=\left[\begin{array}{cc|cccccc}
0 & 3 & 0 & 0 & 0 & 0 & 0 & 0 \\
1 & 1 & 0 & 0 & 0 & 0 & 0 & 0 \\
\hline 0 & 0 & 0 & 0 & 0 & 3 & 0 & 0 \\
0 & 0 & 0 & -\frac{2}{3} & 0 & -\frac{2}{3} & 0 & \frac{1}{3} \\
0 & 0 & 0 & 0 & 0 & 0 & 1 & 0 \\
0 & 0 & 1 & -\frac{2}{3} & 0 & \frac{1}{3} & 0 & -\frac{2}{3} \\
0 & 0 & 0 & 0 & 1 & 0 & 0 & 0 \\
0 & 0 & 0 & \frac{1}{3} & 0 & -\frac{2}{3} & 0 & -\frac{2}{3}
\end{array}\right]
$$

and the Fourier similarity transform $F S T_{\pi}(G)$ of $G$ is shown in Figure 3.2.

Since $P^{-1} F S T_{\pi}(A) P$ is a similarity transform of $A$ (see Equation (3.3)) then it follows that this transform preserves the eigenvalues of the matrix. This gives us the following direct corollary of Theorem 3.2 . 


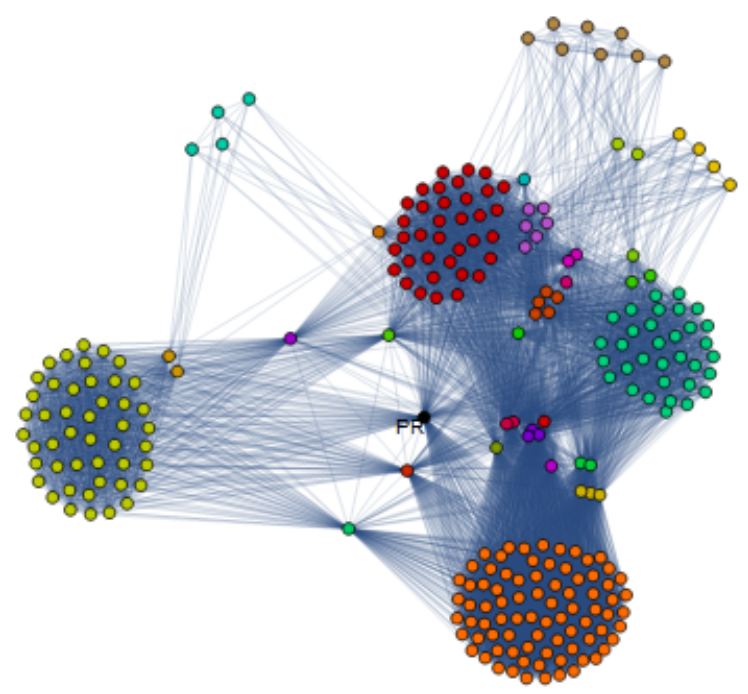

Figure 3.3: A social network of 254 colonists in the Boston, Massachusetts area in the late eighteenth century is shown. Each of the colonists belong to at least one of seven organizations and an edge indicates that two individuals are members of the same organization. Vertices are colored to indicate elements of a specific equitable partition. The single black vertex is the partition element consisting of a single individual, Paul Revere, who notably is a member of five out of seven organizations.

Corollary 3.6. (Graph Spectrum and Fourier Decompositions) Suppose the graph $G$ with adjacency matrix $A$ has the equitable partition $\pi$. If $P^{-1} F S T_{\pi}(A) P=A_{\pi} \oplus B$ then

$$
\sigma(A)=\sigma\left(A_{\pi}\right) \cup \sigma(B)
$$

Note that this gives us an alternate proof of the fact that $\sigma\left(A_{\pi}\right) \subseteq \sigma(A)$ from [9] and [13]. Moreover, via a FST we obtain the matrix $B \in \mathbb{C}^{(n-k) \times(n-k)}$ which has the other eigenvalues of $A$. Also, the eigenvalues of $B$ may also be important to the spectral analysis of the graph $G$ and may be easier to find by analyzing $B$ rather than the larger matrix $A$.

To provide another example, recall from Chapter 2 that nontrivial equitable partitions have been shown to be ubiquitous in real-networks [19]. As such, Figure 3.3 shows a social network of colonists that includes Paul Revere in the Boston, Massachusetts area in the 

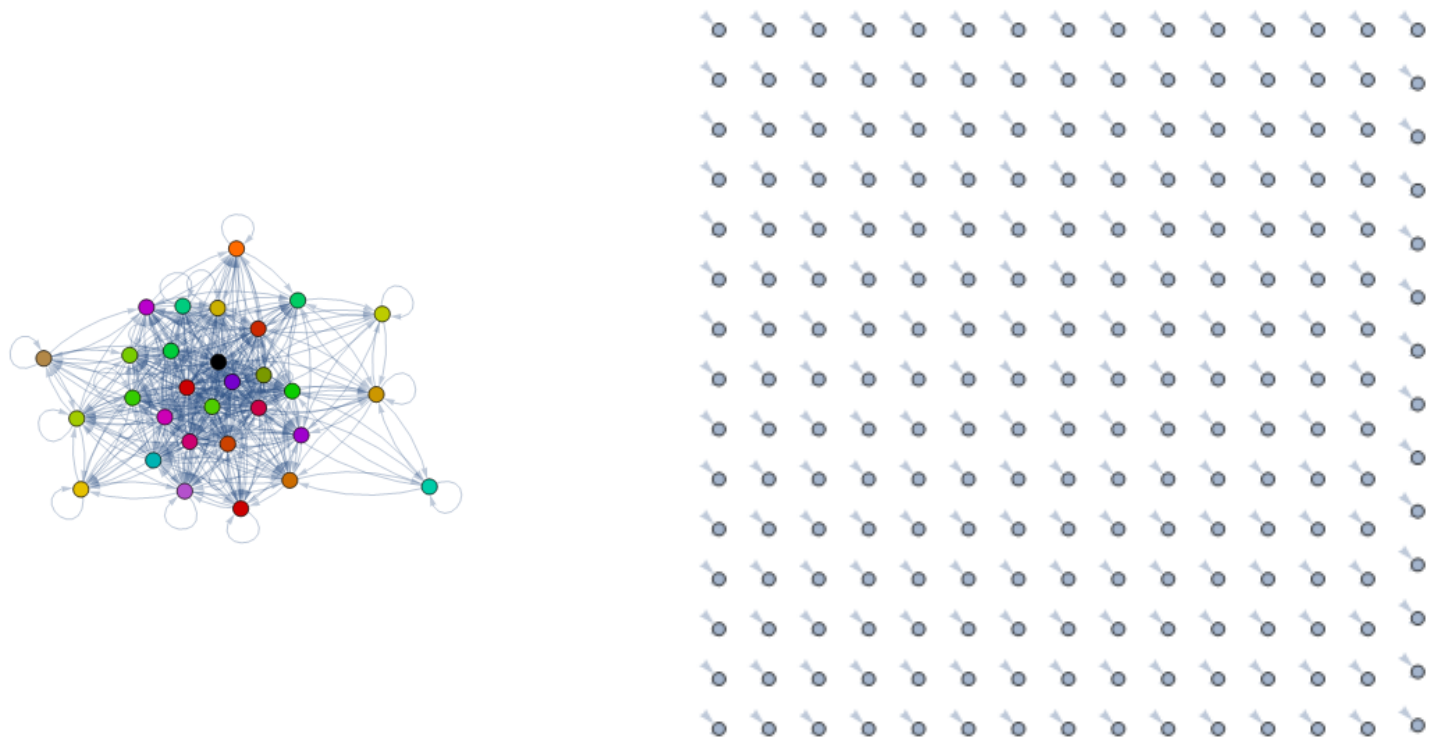

Figure 3.4: The Fourier similarity transform of the Paul Revere social network in Figure 3.3. The divisor graph is on the left with the remainder of the decomposed graph on the right. Each color represents the equitable partition element of the same color shown in Figure 3.3. The black vertex represents the partition element containing the single individual Paul Revere. As in previous figures edge weights are omitted.

late eighteenth century. Here, an edge indicates whether two individuals belong to the same organization. In this figure two individuals are in the same element of the equitable partition if they have the same color. The following figure, Figure 3.4, is the Fourier similarity transform for this network. A feature that will be explored in later works is the fact that this decomposition results in 225 graphs, one that is the divisor graph $G_{\pi}$ consisting of thirty vertices each representing an element of the equitable partition. The other 224 graphs are single vertices with a loop. That is, although Theorem 3.2 guarantees a decomposition into at least two graphs, the Fourier transform can result in a much more complete decomposition of a graph (network). 


\section{Chapter 4. Proof of the Graph Decomposi- TION THEOREM}

In this chapter we give a proof of Theorem 3.2. Before doing so we require the following lemma.

Lemma 4.1. If an undirected graph $G=(V, E, \omega)$ has adjacency matrix $A$ and an equitable partition $\pi=\left\{V_{1}, V_{2}, \ldots, V_{k}\right\}$ such that $\sum_{j \in V_{b}} A_{i j}=D_{a b}$ for any $i \in V_{a}$, then $\sum_{i \in V_{a}} A_{i j}=$ $D_{b a}$ for any $j \in V_{b}$.

Proof. Since $G$ is an undriected graph, then $A=A^{T}$. Thus, for any $j \in V_{b}$

$$
\sum_{i \in V_{a}} A_{i j}=\sum_{i \in V_{a}} A_{j i}=D_{b a}
$$

A useful way to think about Lemma 4.1 is that in an undirected graph every connection from a vertex in $V_{a}$ to a vertex in $V_{b}$ is also a connection from a vertex in $V_{b}$ to a vertex in $V_{a}$. If the partial row sums are equal, then, since $A$ is symmetric, it must also be true that the partial column sums are equal. This is because the partial column sums of $A$ are the partial row sums of $A^{T}=A$. The reason for this lemma will become clear by comparing Theorem 3.2 with Theorem 5.4 where we extend the result of Theorem 3.2 to directed graphs.

With Lemma 4.1 in place we provide a proof of Theorem 3.2, which also gives a proof of Lemma 3.3.

Proof. Let $G=(V, E, \omega)$ be an undirected graph with adjacency matrix $A \in \mathbb{C}^{n \times n}$. Also, let $\pi=\left\{V_{1}, \ldots, V_{k}\right\}$ be a partition of the vertices $V$ of the graph $G$. We also assume the labeling of the graph verticies is consistent with our previous convention. Namely, that if $v_{x} \in V_{i}$ and $v_{y} \in V_{j}$ with $i<j$, then $x<y$ for all $1 \leq x, y \leq n$ and $1 \leq i, j \leq k$. Finally, we also use $\pi$ and $G$ to define $P$ as in Lemma 3.3, and let $\left|V_{i}\right|=n_{i}$. 
Using the notation $A[a, b]$, introduced in Chapter 3 we write $A$ as

$$
A=\left[\begin{array}{ccccc}
A[1,1] & A[1,2] & A[1,3] & \ldots & A[1, k] \\
A[2,1] & A[2,2] & A[2,3] & \ldots & A[2, k] \\
A[3,1] & A[3,2] & A[3,3] & \ldots & A[3, k] \\
\vdots & \vdots & \vdots & \ddots & \vdots \\
A[k, 1] & A[k, 2] & A[k, 3] & \ldots & A[k, k]
\end{array}\right]
$$

where $A[a, b] \in C^{n_{a} \times n_{b}}$ corresponding to the partition $\pi$. We then have

$$
F S T_{\pi}(A)=W_{\pi}^{-1} A W_{\pi}=\left[\begin{array}{cccc}
\frac{1}{n_{1}} W_{n_{1}}^{H} A[1,1] W_{n_{1}} & \frac{1}{n_{1}} W_{n_{1}}^{H} A[1,2] W_{n_{2}} & \ldots & \frac{1}{n_{1}} W_{n_{1}}^{H} A[1, k] W_{n_{k}} \\
\frac{1}{n_{2}} W_{n_{2}}^{H} A[2,1] W_{n_{1}} & \frac{1}{n_{2}} W_{n_{2}}^{H} A[2,2] W_{n_{2}} & \ldots & \frac{1}{n_{2}} W_{n_{2}}^{H} A[2, k] W_{n_{k}} \\
\vdots & \vdots & \ddots & \vdots \\
\frac{1}{n_{k}} W_{n_{k}}^{H} A[k, 1] W_{n_{1}} & \frac{1}{n_{k}} W_{n_{k}}^{H} A[k, 2] W_{n_{2}} & \cdots & \frac{1}{n_{k}} W_{n_{k}}^{H} A[k, k] W_{n_{k}}
\end{array}\right]
$$

in which $\operatorname{FST}_{\pi}(A)[a, b]=\frac{1}{n_{a}} W_{n_{a}}^{H} A[a, b] W_{n_{b}}$.

Note that the permutation matrix $P$ described in Lemma 3.3 takes the first row and column of each block $\frac{1}{n_{a}} W_{n_{a}}^{H} A[a, b] W_{n_{b}}$ and moves it to the $a^{\text {th }}$ row and $b^{\text {th }}$ column (see Remark 3). Hence, the statement

$$
P^{-1} F_{S T}(A) P=\tilde{A} \oplus B
$$

is equivalent to saying that for each block $\frac{1}{n_{a}} W_{n_{a}}^{H} A[a, b] W_{n_{b}}$, the top left entry is some constant (for now, we will use a placeholder value dependent on $a$ and $b$, called $\tilde{a}_{a b}$ ), and every other entry in either the first row or first column is 0 . That is,

$$
\left[\frac{1}{n_{a}} W_{n_{a}}^{H} A[a, b] W_{n_{b}}\right]_{x y}= \begin{cases}\tilde{a}_{a b} & \text { for } x=y=1 \\ 0 & \text { for } x \neq y=1 \text { or } y \neq x=1 .\end{cases}
$$

Our goal is to show that Equation (4.2) holds if and only if $\pi$ is an equitable partition. 
Assume then that $\pi$ is an equitable partition. Then the partial row sum $\sum_{j \in V_{b}} A_{i j}=D_{a b}$ is constant for any $i \in V_{a}$ with $a$ fixed. Thus,

$$
\left[\frac{1}{n_{a}} W_{n_{a}}^{H} A[a, b] W_{n_{b}}\right]_{x y}=\frac{1}{n_{a}} \sum_{s=1}^{n_{a}} \sum_{t=1}^{n_{b}} c_{s t} \bar{\omega}_{n_{a}}^{(x-1)(s-1)} \omega_{n_{b}}^{(y-1)(t-1)}
$$

where $[A[a, b]]_{s t}=c_{s t}$. When $x=y=1$ we have

$$
\left[\frac{1}{n_{a}} W_{n_{a}}^{H} A[a, b] W_{n_{b}}\right]_{11}=\frac{1}{n_{a}} \sum_{s=1}^{n_{a}} \sum_{t=1}^{n_{b}} c_{s t} \bar{\omega}_{n_{a}}^{0} \omega_{n_{b}}^{0}=\frac{1}{n_{a}} \sum_{s=1}^{n_{a}} \sum_{t=1}^{n_{b}} c_{s t} .
$$

Because $\pi$ is an equitable partition, our labeling assumption gives us that every $s$ involved in this sum relates to a vertex $v_{i} \in V_{a}$. Thus,

$$
\sum_{t=1}^{n_{b}} c_{s t}=\sum_{t=1}^{n_{b}}[A[a, b]]_{s t}=\sum_{j \in V_{b}} A_{i j}=D_{a b}
$$

for every choice of $i \in V_{a}$, or rather, for each $s$. Thus

$$
\left[\frac{1}{n_{a}} W_{n_{a}}^{H} A[a, b] W_{n_{b}}\right]_{11}=\frac{1}{n_{a}} \sum_{s=1}^{n_{a}}\left(\sum_{t=1}^{n_{b}} c_{s t}\right)=\frac{1}{n_{a}} \sum_{s=1}^{n_{a}} D_{a b}=D_{a b}
$$

so that Equation (4.2) holds in the case that $x=y=1$.

In the case that $x \neq 1$, and $y=1$ we have,

$$
\begin{aligned}
{\left[\frac{1}{n_{a}} W_{n_{a}}^{H} A[a, b] W_{n_{b}}\right]_{x 1}=} & \frac{1}{n_{a}} \sum_{s=1}^{n_{a}} \sum_{t=1}^{n_{b}} c_{s t} \bar{\omega}_{n_{a}}^{(x-1)(s-1)} \omega_{n_{b}}^{0}=\frac{1}{n_{a}} \sum_{s=1}^{n_{a}} \sum_{t=1}^{n_{b}} c_{s t} \bar{\omega}_{n_{a}}^{(x-1)(s-1)} \\
& =\frac{1}{n_{a}} \sum_{s=1}^{n_{a}} \bar{\omega}_{n_{a}}^{(x-1)(s-1)}\left(\sum_{t=1}^{n_{b}} c_{s t}\right) \\
& =\left(\frac{D_{a b}}{n_{a}}\right) \sum_{s=1}^{n_{a}} \bar{\omega}_{n_{a}}^{(x-1)(s-1)} \text { (by Definition 2.2) } \\
& =\left(\frac{D_{a b}}{n_{a}}\right)(0) \\
& =0 .
\end{aligned}
$$


When we look at the $x=1$ and $y \neq 1$ case, we get

$$
\begin{aligned}
{\left[\frac{1}{n_{a}} W_{n_{a}}^{H} A[a, b] W_{n_{b}}\right]_{1 y}=} & \frac{1}{n_{a}} \sum_{s=1}^{n_{a}} \sum_{t=1}^{n_{b}} c_{s t} \bar{\omega}_{n_{a}}^{0} \omega_{n_{b}}^{(y-1)(t-1)}=\frac{1}{n_{a}} \sum_{s=1}^{n_{a}} \sum_{t=1}^{n_{b}} c_{s t} \omega_{n_{b}}^{(y-1)(t-1)} \\
& =\frac{1}{n_{a}} \sum_{t=1}^{n_{b}} \omega_{n_{b}}^{(y-1)(t-1)}\left(\sum_{s=1}^{n_{a}} c_{s t}\right) \\
& =\left(\frac{D_{b a}}{n_{a}}\right) \sum_{t=1}^{n_{b}} \omega_{n_{b}}^{(y-1)(t-1)} \text { (by Lemma 4.1) } \\
& =\left(\frac{D_{b a}}{n_{a}}\right)(0) \\
& =0 .
\end{aligned}
$$

These cases together imply that Equation (4.2) holds when either $x \neq 1$ and $y=1$ or $x=1$ and $y \neq 1$. Hence, Equation (4.2) holds implying $P^{-1} F S T_{\pi}(A) P=A_{\pi} \oplus B$ as desired.

Conversely, suppose now that $P^{-1} F S T_{\pi}(A) P=\tilde{A} \oplus B$ for the vertex partition $\pi$, or rather that the matrix $F S T_{\pi}(A)=W_{\pi}^{-1} A W_{\pi}$ has the property that

$$
\left[\frac{1}{n_{a}} W_{n_{a}}^{H} A[a, b] W_{n_{b}}\right]_{x y}= \begin{cases}\tilde{a}_{a b} & \text { for } x=y=1 \\ 0 & \text { for } x \neq y=1 \text { or } y \neq x=1\end{cases}
$$

where again, $\tilde{a}_{a b}$ is some placeholder value dependent on $a$ and $b$.

In order to consider the first column of this block of $F S T_{\pi}(A)$, we calculate $\frac{1}{n_{a}} W_{n_{a}}^{H} A[a, b] \mathbb{1}_{n_{b}}$, where $\mathbb{1}_{n_{b}}$ is the vector of all ones of length $n_{b}$. This is the scalar $\frac{1}{n_{a}}$ times the row sums of $W_{n_{a}}^{H} A[a, b]=H$. An arbitrary element of $H$ is then calculated to be $H_{x y}=$ $\sum_{s=1}^{n_{a}} \bar{\omega}_{n_{a}}^{(x-1)(s-1)} c_{s y}$, and thus the $x^{t h}$ row sum of $H$ is

$$
\sum_{t=1}^{n_{b}}\left(\sum_{s=1}^{n_{a}} \bar{\omega}_{n_{a}}^{(x-1)(s-1)} c_{s t}\right)=\sum_{s=1}^{n_{a}}\left[\bar{\omega}_{n_{a}}^{(x-1)(s-1)}\left(\sum_{t=1}^{n_{b}} c_{s t}\right)\right]=\sum_{s=1}^{n_{a}}\left[\bar{\omega}_{n_{a}}^{(x-1)(s-1)} R_{s}\right]
$$

where $R_{s}=\sum_{t=1}^{n_{b}} c_{s t}=\sum_{j \in V_{b}} A_{i j}$ for all $i \in V_{a}$ is the partial row sum of $A$. This is equivalent 
to the statement that

$$
\sum_{t=1}^{n_{b}} H_{x t}=\sum_{s=1}^{n_{a}}\left[\bar{\omega}_{n_{a}}^{(x-1)(s-1)} R_{s}\right]=0
$$

for $x=2, \ldots, n_{a}$, which can be written in matrix form as

$$
Z \mathbf{c}=\left[\begin{array}{cccc}
\omega_{n_{a}}^{0} & \omega_{n_{a}}^{-1} & \ldots & \omega_{n_{a}}^{-\left(n_{a}-1\right)} \\
\omega_{n_{a}}^{0} & \omega_{n_{a}}^{-2} & \ldots & \omega_{n_{a}}^{-2\left(n_{a}-1\right)} \\
\vdots & \vdots & \ddots & \vdots \\
\omega_{n_{a}}^{0} & \omega_{n_{a}}^{-\left(n_{a}-1\right)} & \ldots & \omega_{n_{a}}^{-\left(n_{a}-1\right)^{2}}
\end{array}\right]\left[\begin{array}{c}
R_{1} \\
R_{2} \\
\vdots \\
R_{n_{a}}
\end{array}\right]=\mathbf{0}
$$

Note that $Z$ is the inverse of the $n_{a} \times n_{a}$ discrete Fourier transform $W_{n_{a}}$ with the first row removed. This means that the dimension of the row space of $Z$ is $n_{a}-1$ implying that the null space of $Z$ has dimension 1 . Observe that $\mathbf{c}=\mathbb{1}_{n_{a}}$ is in the null space of $Z$, and as such $\operatorname{Null}(Z)=q \mathbb{1}_{n_{a}}$ for $q \in \mathbb{R}$, implying $R_{i}=R_{j}$ for all $1 \leq i, j \leq n_{a}$, so $\sum_{j \in V_{b}} A_{i j}$ is constant $\forall i \in V_{A}$. Hence, the partial row sums of the adjacency matrix corresponding to $\pi$ are equal and $D_{a b}=\frac{1}{n_{a}} \sum_{i \in V_{a}}\left(\sum_{j \in V_{b}} A_{i j}\right)=\sum_{j \in V_{b}} A_{i j}=R_{l}$ for all $1 \leq l \leq n_{a}$, making $\pi$ an equitable partition.

Remark. The fact that the first row of each $A[a, b]$ block, except for the row's first element, is 0 is not explicitly used in either direction of the proof. However, since we are dealing with only undirected graphs at this stage, our condition that the first column of $A[a, b]$ (excepting its first element) is 0 necessarily means that the first row of $A[a, b]$ follow the same pattern. This detail is explained further in Chapter 5 where Theorem 3.2 is extended to directed graphs. 


\section{Chapter 5. Generalization of Equitable De- Compositions to Directed Graphs and}

\section{Consequences}

Our previous definition of an equitable partition was specific to undirected graphs (see Definition 2.2). To extend this concept to directed graphs we define two new types of equitable partitions. Again, for convenience, we define two values to assist us.

Definition 5.1. Let $G=(V, E, \omega)$ be a directed graph with partition $\pi=\left\{V_{1}, V_{2}, \ldots, V_{k}\right\}$ of the vertices $V$. Define

$$
R_{a b}=\frac{1}{n_{a}} \sum_{i \in V_{a}}\left(\sum_{j \in V_{b}} A_{i j}\right)
$$

and

$$
T_{a b}=\frac{1}{n_{b}} \sum_{j \in V_{b}}\left(\frac{n_{b}}{n_{a}} \sum_{i \in V_{a}} A_{i j}\right)=\frac{1}{n_{a}} \sum_{j \in V_{b}}\left(\sum_{i \in V_{a}} A_{i j}\right)
$$

Note that $R_{a b}$ and $T_{a b}$ are anologues to $D_{a b}$ but for directed graphs.

Definition 5.2 (Equitable Receiving Partition). An equitable receiving partition (ERP) of a graph $G=(V, E, \omega)$ is a partition $\pi=\left\{V_{1}, V_{2}, \ldots, V_{k}\right\}$ of the vertices $V$ with the property that for all $a, b \in\{1,2, \ldots, k\}$ the sum

$$
\sum_{j \in V_{b}} A_{i j}=R_{a b}
$$

is constant for any $i \in V_{a}$. The matrix $A_{\pi}=R$ is the (receiving) divisor matrix of $A$ with respect to $\pi$ and $G_{\pi}$ is the (receiving) divisor graph with adjacency matrix $A_{\pi}$.

Definition 5.2 is, in fact, the same as our original definition of an equitable partition with the exception that an ERP is not restricted only to undirected graphs. If a graph $G$ has an ERP then the partial row sums of its adjacency matrix within each partition element is constant. For the graph $G$ this means that every vertex $i \in V_{a}$ receives the same total input, i.e. sum of weights, from any other partition element $V_{b}$. 
Definition 5.3 (Equitable Transmitting Partition). An equitable transmitting partition (ETP) of a graph $G=(V, E, \omega)$ is a partition $\pi=\left\{V_{1}, V_{2}, \ldots, V_{k}\right\}$ of the vertices $V$ with the property that for all $a, b \in\{1,2, \ldots, k\}$ the sum

$$
\sum_{i \in V_{a}} A_{i j}=\frac{n_{a}}{n_{b}} T_{a b}
$$

is constant for any $j \in V_{b}$. The matrix $A_{\pi}=T$ is the (transmitting) divisor matrix of $A$ with respect to $\pi$ and $G_{\pi}$ is the (transmitting) divisor graph with adjacency matrix $A_{\pi}$.

The difference between an ETP and an ERP is that an ERP has constant partial row sums within partition elements, while the ETP has constant partial column sums. For the graph $G$ this is equivalent to saying that every vertex $j \in V_{b}$ sends the same total output, i.e. sum of weights, to any other partition element $V_{a}$.

We note that if an undirected graph $G$ has an equitable partition $\pi$, as defined in Chapter 2, then $\pi$ is both an ERP and an ETP if we consider $G$ to be a directed graph, i.e. each of the graphs edges is replaced with two directed edges oriented in opposite directions. However, the converse is not always true. A partition can be both an ERP and an ETP but not an equitable partition of an undirected graph (see Example 5.5). 
Theorem 5.4. (Fourier Decompositions of Directed Graphs) Let $G=(V, E, \omega)$ be a directed graph with vertex partition $\pi=\left\{V_{1}, V_{2}, \ldots, V_{k}\right\}$. We can construct a permutation matrix $P \in\{0,1\}^{n \times n}$ such that the permuted discrete Fourier transform of $A=A(G)$ decomposes into the direct product

$$
P^{-1} F S T_{\pi}(A) P=A_{\pi} \oplus B \in \mathbb{C}^{n \times n}
$$

if and only if $\pi$ is both an ERP and an ETP of $G$. Additionally, the matrix $F S T_{\pi}(A)$ is reducible if and only if $\pi$ is either an ERP or an ETP. Specifically, we can construct a permutation matrix $P$ such that

$$
P^{-1} F_{\pi}(A) P=\left[\begin{array}{cc}
A_{\pi} & C \\
0 & B
\end{array}\right]
$$

if and only if $\pi$ is an ERP, and

$$
P^{-1} F_{S}(A) P=\left[\begin{array}{cc}
A_{\pi} & 0 \\
C & B
\end{array}\right]
$$

if and only if $\pi$ is an ETP. We refer to this as a weak decomposition of $A$ since the associated graph has at least two weakly connected components corresponding to $A_{\pi}$ and $B$, respectively.

Remark. The permutation matrix $P$ in Theorem 5.4 is the same permutation matrix constructed for Theorem 3.2, defined in Lemma 3.3.

Proof. First, we note that proving

$$
P^{-1} F S T_{\pi}(A) P=\left[\begin{array}{cc}
A_{\pi} & C \\
0 & B
\end{array}\right]
$$


if and only if $\pi$ is an ERP and

$$
P^{-1} F S T_{\pi}(A) P=\left[\begin{array}{cc}
A_{\pi} & 0 \\
C & B
\end{array}\right]
$$

if and only if $\pi$ is an ETP necessarily proves that

$$
P^{-1} F S T_{\pi}(A) P=A_{\pi} \oplus B
$$

if and only if $\pi$ is both an ERP and an ETP. As such, we focus on proving Equations (5.1) and $(5.2)$

Suppose then that $\pi$ is an ERP. Note that the definition of an ERP is the same as the definition of an equitable partition for an undirected graph (see Definition 2.2) where $R_{a b}=D_{a b}$ for a directed graph. However, the proof of Theorem 3.2 in the specific case that $x \neq 1$ and $y=1$ does not rely on the fact that $G$ is directed Hence, the argument in the proof of Theorem 3.2 also proves that Equation (5.1) holds if and only if $\pi$ is an ERP.

To prove Equation (5.2) holds if and only if $\pi$ is an ETP we suppose for the moment that $\pi$ is an ETP. To prove Equation (5.2) holds we then need to show

$$
\left[\frac{1}{n_{a}} W_{n_{a}}^{H} A[a, b] W_{n_{b}}\right]_{x y}= \begin{cases}T_{a b} & \text { for } x=y=1 \\ 0 & y \neq x=1\end{cases}
$$

If $x=y=1$ then

$$
\left[\frac{1}{n_{a}} W_{n_{a}}^{H} A[a, b] W_{n_{b}}\right]_{11}=\frac{1}{n_{a}} \sum_{s=1}^{n_{a}} \sum_{t=1}^{n_{b}} c_{s t}=\frac{1}{n_{a}} \sum_{t=1}^{n_{b}} \sum_{s=1}^{n_{a}} c_{s t}
$$

as in Equation (4.3). Since $\pi$ is an ETP then

$$
\sum_{s=1}^{n_{a}} c_{s t}=\sum_{i \in V_{a}} A_{i j}=\frac{n_{a}}{n_{b}} T_{a b}
$$


so that

$$
\frac{1}{n_{a}} \sum_{t=1}^{n_{b}} \sum_{s=1}^{n_{a}} c_{s t}=\frac{1}{n_{a}} \sum_{t=1}^{n_{b}}\left(\frac{n_{a}}{n_{b}} T_{a b}\right)=\frac{1}{n_{b}} \sum_{t=1}^{n_{b}} T_{a b}=T_{a b}
$$

Now, we look specifically at the $x=1$ and $y \neq 1$ case. The argument is exactly the one presented in the proof of Theorem 3.2, except it utilizes Definition 5.3 instead of Lemma 4.1. Thus, if $\pi$ is an equitable partition then Equation (5.2) holds.

Now assume that Equation (5.2) holds, so that the $F S T_{\pi}(A)$ is reducible via the permutation matrix $P$ defined in Lemma 3.3. The argument that $\pi$ must be an ETP is very similar to the one provided in the proof of Theorem 3.2. However, there are minor details that are different. Specifically, in order to consider the first row of $\frac{1}{n_{a}} W_{n_{a}}^{H} A[a, b] W_{n_{b}}$ we can look at $\frac{1}{n_{a}} \mathbb{1}_{n_{a}}^{T} A[a, b] W_{n_{b}}$, which is a scalar times the column sums of $A[a, b] W_{n_{b}}=F$. Using the same notation as before, an arbitrary element of $F$ is calculated as

$$
F_{x y}=\sum_{t=1}^{n_{b}} c_{x t} \omega_{n_{b}}^{(t-1)(y-1)}
$$

and thus the $y^{\text {th }}$ column sum of $F$ is

$$
\sum_{s=1}^{n_{a}}\left(\sum_{t=1}^{n_{b}} c_{s t} \omega_{n_{b}}^{(t-1)(y-1)}\right)=\sum_{t=1}^{n_{b}}\left[\omega_{n_{b}}^{(t-1)(y-1)}\left(\sum_{s=1}^{n_{a}} c_{s t}\right)\right]=\sum_{t=1}^{n_{b}}\left[\omega_{n_{b}}^{(t-1)(y-1)} C_{t}\right]
$$

where $C_{t}=\sum_{s=1}^{n_{a}} c_{s t}=\sum_{i \in V_{a}} A_{i j}$ for all $j \in V_{b}$, or rather, for every $t$. Equation 5.2 then gives

$$
\sum_{s=1}^{n_{a}} F_{s y}=\sum_{t=1}^{n_{b}}\left[\omega_{n_{b}}^{(t-1)(y-1)} C_{t}\right]=0
$$

for $y=2, \ldots, n_{b}$. Which we write as a system of equations in matrix form as

$$
Z \mathbf{c}=\left[\begin{array}{cccc}
\omega_{n_{b}}^{0} & \omega_{n_{b}}^{1} & \ldots & \omega_{n_{b}}^{n_{b}-1} \\
\omega_{n_{b}}^{0} & \omega_{n_{b}}^{2} & \ldots & \omega_{n_{b}}^{2\left(n_{b}-1\right)} \\
\vdots & \vdots & \ddots & \vdots \\
\omega_{n_{b}}^{0} & \omega_{n_{b}}^{n_{b}-1} & \ldots & \omega_{n_{b}}^{\left(n_{b}-1\right)^{2}}
\end{array}\right]\left[\begin{array}{c}
C_{1} \\
C_{2} \\
\vdots \\
C_{n_{b}}
\end{array}\right]=\mathbf{0}
$$


Similar to before, $Z$ is the $n_{b} \times n_{b}$ discrete Fourier transform with the first row removed so the rows of $Z$ are linearly independent. Thus the row space of $Z$ has dimension $n_{b}-1$ implying that the null space of $Z$ has dimension 1 . Once again, $\mathbf{c}=\mathbb{1}_{n_{b}} \in \operatorname{Null}(Z)$, and so $\operatorname{Null}(Z)=q \mathbb{1}_{m}$ for $q \in \mathbb{R}$, which implies that $C_{i}=C_{j}$ for all $1 \leq i, j \leq n_{b}$, so $\sum_{i \in V_{a}} A_{i j}$ is constant for all $j \in V_{b}$. Hence, the partial column sums of the adjacency matrix corresponding to $\pi$ are equal making $\pi$ an equitable transmitting partition.

Example 5.5. Consider the graph $G$ with the adjacency matrix

$$
A=\left[\begin{array}{ll|llllll}
0 & 0 & 1 & 1 & 1 & 0 & 0 & 0 \\
0 & 0 & 0 & 0 & 0 & 1 & 1 & 1 \\
\hline 0 & 1 & 0 & 0 & 0 & 1 & 0 & 0 \\
1 & 0 & 0 & 0 & 1 & 0 & 0 & 0 \\
1 & 0 & 0 & 1 & 0 & 0 & 0 & 0 \\
1 & 0 & 1 & 0 & 0 & 0 & 0 & 0 \\
0 & 1 & 0 & 0 & 0 & 0 & 0 & 1 \\
0 & 1 & 0 & 0 & 0 & 0 & 1 & 0
\end{array}\right]
$$

shown in Figure 5.1 (left). The graph has the partition $\pi=\{\{1,2\},\{3,4,5,6,7,8\}\}$ which is both an ETP and ERP although not an equitable partition as defined in Definition 2.2 as $G$ is a directed graph. The matrix $W_{\pi}$ is again

$$
W_{\pi}=\left[\begin{array}{cc}
W_{2} & 0 \\
0 & W_{6}
\end{array}\right] \in \mathbb{C}^{8 \times 8}
$$

and the Fourier similarity transform of the matrix $A$ is given by the matrix 

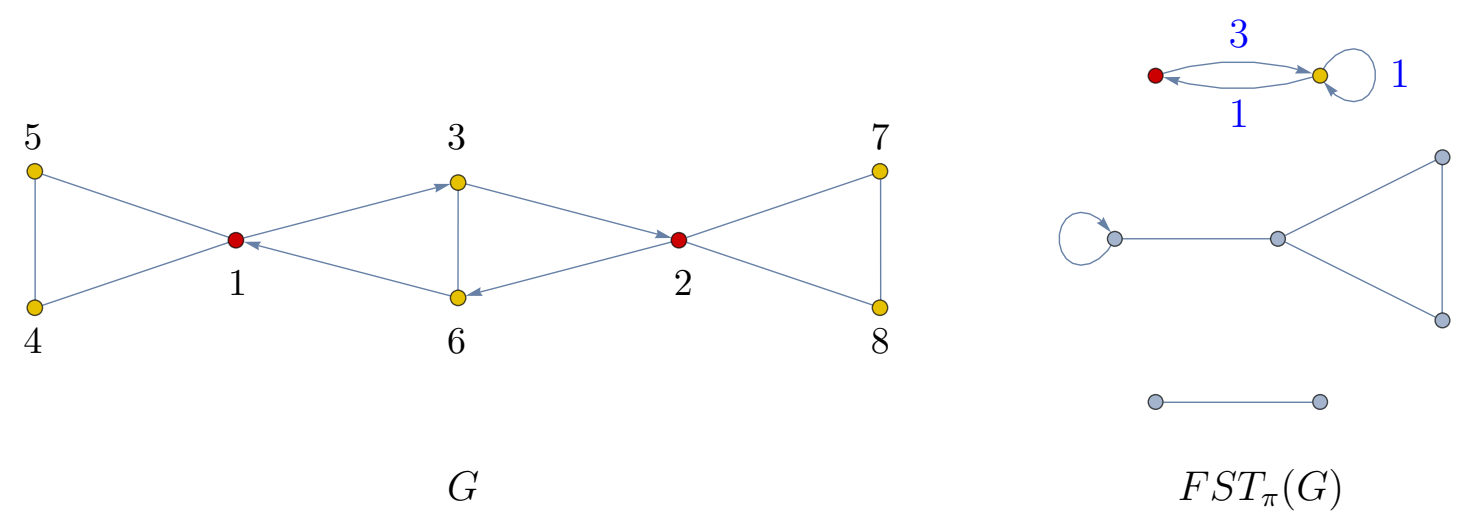

$F S T_{\pi}(G)$

Figure 5.1: The unweighted but directed graph $G$ (left) has the partition $\pi=$ $\{\{1,2\},\{3,4,5,6,7,8\}\}$ which is both an ETP and an ERP but not an equitable partition. The Fourier similarity transform $F S T_{\pi}(G)$ is shown (right) with edges weights omitted except for those of the divisor graph.

$$
F_{S T}(A)=\left[\begin{array}{cc|cccccc}
0 & 0 & 3 & 0 & 0 & 0 & 0 & 0 \\
0 & 0 & 0 & -1+\sqrt{3} i & 0 & -1 & 0 & -1-\sqrt{3} i \\
\hline 1 & 0 & 1 & 0 & 0 & 0 & 0 & 0 \\
0 & \frac{1}{3}(1-\sqrt{3} i) & 0 & 0 & 0 & 0 & 0 & -1 \\
0 & 0 & 0 & 0 & 0 & 0 & 1 & 0 \\
0 & \frac{1}{3} & 0 & 0 & 0 & -1 & 0 & 0 \\
0 & 0 & 0 & 0 & 1 & 0 & 0 & 0 \\
0 & \frac{1}{3}(1+\sqrt{3} i) & 0 & -1 & 0 & 0 & 0 & 0
\end{array}\right] .
$$

The permutation matrix $P$ is

$$
P=\left[\begin{array}{llllllll}
\mathbf{e}_{1} & \mathbf{e}_{3} & \mathbf{e}_{2} & \mathbf{e}_{4} & \mathbf{e}_{5} & \mathbf{e}_{6} & \mathbf{e}_{7} & \mathbf{e}_{8}
\end{array}\right]
$$

which is found by using Lemma 3.1 and the permuted similarity transform is 


$$
P^{-1} F S T_{\pi}(A) P=\left[\begin{array}{cc|cccccc}
0 & 3 & 0 & 0 & 0 & 0 & 0 & 0 \\
1 & 1 & 0 & 0 & 0 & 0 & 0 & 0 \\
\hline 0 & 0 & 0 & -1+\sqrt{3} i & 0 & 1 & 0 & -1-\sqrt{3} i \\
0 & 0 & \frac{1}{3}(1-\sqrt{3} i) & 0 & 0 & 0 & 0 & -1 \\
0 & 0 & 0 & 0 & 0 & 0 & 1 & 0 \\
0 & 0 & \frac{1}{3} & 0 & 0 & -1 & 0 & 0 \\
0 & 0 & 0 & 0 & 1 & 0 & 0 & 0 \\
0 & 0 & \frac{1}{3}(1+\sqrt{3} i) & -1 & 0 & 0 & 0 & 0
\end{array}\right] .
$$

The Fourier similarity transform $F S T_{\pi}(G)$ of $G$ is shown in Figure 5.1 (right). Note that, as guaranteed by Theorem 5.4, since $\pi$ is both an ERP and an ETP the matrix $A$ decomposes into the direct sum $A=A_{\pi} \oplus B$.

To demonstrate what happens when a matrix has a partition that is not both an ERP and an ETP but only one of these we consider the following example.

Example 5.6. Let the graph $G$ have the adjacency matrix

$$
A(G)=\left[\begin{array}{ll|llllll}
0 & 0 & 1 & 0 & 1 & 1 & 0 & 0 \\
0 & 0 & 0 & 1 & 0 & 0 & 1 & 1 \\
\hline 1 & 0 & 0 & 0 & 0 & 1 & 0 & 0 \\
0 & 1 & 0 & 0 & 0 & 0 & 1 & 0 \\
0 & 0 & 0 & 0 & 0 & 0 & 0 & 1 \\
0 & 0 & 1 & 0 & 0 & 0 & 0 & 0 \\
0 & 0 & 0 & 1 & 0 & 0 & 0 & 0 \\
0 & 0 & 0 & 0 & 1 & 0 & 0 & 0
\end{array}\right] .
$$

Then the partition $\pi=\{\{1,2\},\{3,4,5,6,7,8\}\}$ is an ETP but not an ERP. Using the same 
permutation matrix $P$ as in Example 5.5 the permuted similarity transform is

$$
P^{-1} F S T_{\pi}(A) P=\left[\begin{array}{cc|cccccc}
0 & 3 & 0 & 0 & 0 & 0 & 0 & 0 \\
1 & 1 & 0 & 0 & 0 & 0 & 0 & 0 \\
\hline 0 & 0 & 0 & -\frac{1}{2} \alpha_{-} & \frac{1}{2} \beta_{-} & 1 & 0 & 1 \\
\frac{1}{12} \beta_{-} & 0 & \frac{1}{12} \alpha_{+} & -1 & 0 & 0 & 0 & 0 \\
\frac{1}{12} \alpha_{-} & 0 & \frac{1}{12} \beta_{+} & 0 & 1 & 0 & 0 & 0 \\
0 & 0 & \frac{1}{3} & 0 & 0 & -1 & 0 & 0 \\
\frac{1}{12} \beta_{+} & 0 & \frac{1}{12} \alpha_{-} & 0 & 0 & 0 & 0 & -1 \\
\frac{1}{12} \beta_{+} & \frac{1}{12} \alpha_{-} & 0 & 0 & 0 & 0 & -1 &
\end{array}\right]
$$

where $\alpha_{ \pm}=1 \pm \sqrt{3} i$ and $\beta_{ \pm}=3 \pm \sqrt{3} i$, which is block lower-triangular as guaranteed by Theorem 5.4.

Strictly speaking the Fourier transform in Example 5.6 is not a decomposition of the matrix $A$ into the direct product of other matrices. However, the resulting matrix has the same eigenvalues as the original and as the eigenvalues of an upper and lower block-triangular matrix are the eigenvalues of the respective diagonal blocks the following holds (cf. Corollary 3.6).

Corollary 5.7. (Graph Spectrum, ERPs, and, ETPs) Suppose the graph G with adjacency matrix $A$ has the partition $\pi$. If $\pi$ is an ERP where

$$
P^{-1} F_{S T}(A) P=\left[\begin{array}{cc}
A_{\pi} & C \\
0 & B
\end{array}\right]
$$

then $\sigma(A)=\sigma\left(A_{\pi}\right) \cup \sigma(B)$. Similarly, if $\pi$ is an ETP where

$$
P^{-1} F S T(A) P=\left[\begin{array}{ll}
A_{\pi} & 0 \\
C & B
\end{array}\right]
$$


then $\sigma(A)=\sigma\left(A_{\pi}\right) \cup \sigma(B)$.

Note that, as with standard equitable partitions, this shows that the eigenvalues of the divisor matrix of an ERP and an ETP are a subset of the adjacency matrix $A$, i.e. $\sigma\left(A_{\pi}\right) \subset$ $\sigma(A)$.

\section{Chapter 6. Fourier Decompositions over SYMMETRIES}

A special case of an equitable partition is a graph symmetry. In a simple graph $G=(V, E)$ a symmetry is a permutation $\phi: V \rightarrow V$ of the graph's vertices that preserves adjacencies, i.e. preserves each vertex' neighbors. The permutation $\phi$ is called an automorphism of $G$ and the symmetries of the graph $G$ are the graph's set of automorphisms. More intuitively, an automorphism describes how parts of a graph can be interchanged in a way that preserves the graph's overall structure. In this sense these parts, i.e., subgraphs, are symmetrical and together constitute a graph symmetry.

In general, an automorphism of a graph $G=(V, E, \omega)$ can be defined as follows.

Definition 6.1. (Graph Automorphism) An automorphism $\phi$ of a graph $G=(V, E, \omega)$ is a permutation of $V$ such that the adjacency matrix $A=A(G)$ satisfies

$$
A_{i j}=A_{\phi(i) \phi(j)}
$$

for each pair of vertices $i$ and $j$. The set of all automorphisms of $G$ is a group, denoted by $\operatorname{Aut}(G)$. The order of $\phi$ is the smallest positive integer $\ell=|\phi|$ such that $\phi^{\ell}$ is the identity.

As it turns out, every graph symmetry gives us an equitable partition. The reason is that given an automorphism $\phi$ of the graph the orbit of a vertex $v \in V$ is the vertex set

$$
\mathcal{O}(v)=\left\{\phi^{i}(v): i=1,2, \ldots, \ell \text { for } \ell=|\phi|\right\}
$$



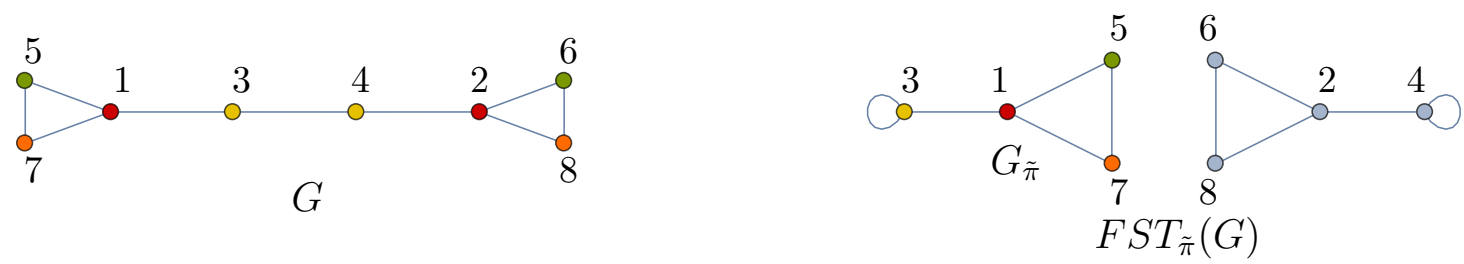

Figure 6.1: The Fourier graph decomposition $F S T_{\tilde{\pi}}(G)$, shown right, is the decomposition of the simple graph $G$, shown left, over the equitable partition $\tilde{\pi}=\{\{1,2\},\{3,4\},\{5,6\},\{7,8\}\}$. The subgraph $G_{\tilde{\pi}}$ of the decomposition is the left half of $F S T_{\tilde{\pi}}(G)$. Edge weights of the graph are omitted.

The orbits of the graph's vertices under $\phi$ collectively partition the graph's vertices into an equitable partition.

Example 6.2. (Symmetries as Equitable Partitions) For instance, the graph $G$ in Figure 6.1 (left), which is also considered in Figure 2.1, has the automorphism $\phi=(12)(34)(56)(78)$ meaning that $\phi(1)=2, \phi(2)=1$, and so on. The orbits of $\phi$ are the sets

$$
V_{1}=\{1,2\}, V_{2}=\{3,4\}, V_{3}=\{5,6\}, V_{4}=\{7,8\}
$$

which yields the equitable partition $\tilde{\pi}=\{\{1,2\},\{3,4\},\{5,6\},\{7,8\}\}$. The associated Fourier decomposition $F S T_{\tilde{\pi}}(G)$ shown in Figure 6.1 (right).

However, not all equitable partitions are generated by symmetries. The equitable partition

$$
\pi=\{\{1,2\},\{3,4,5,6,7,8\}\}
$$

in Example 3.5 is an example of an equitable partition that is not induced by a graph symmetry. The reason is that none of the vertices $3,4,5$, or 6 are symmetric with the vertices 7 or 8 , although both are in the same element of $\pi$.

Previously, it has been shown that it is possible to decompose a graph $G$ with respect to any automorphism $\phi \in \operatorname{Aut}(G)$ similar to what is done in Theorem 3.2. Specifically, if $\phi$ is an automorphism of $G$ then it is possible to construct an invertible matrix $S \in \mathbb{R}^{n \times n}$ such 
that

$$
S A S^{-1}=A_{\phi} \oplus B
$$

where $A_{\phi}$ is the divisor matrix corresponding to the equitable partition induced by $\phi$. The decompositions given by (6.1) are referred to as equitable decompositions and are described in a sequence of papers (see $[2,10,11])$.

Initially, the theory of equitable decompositions was limited to decompositions over uniform automorphisms or automorphisms in which each nontrivial orbit has the same fixed size [2] (cf. Example 6.2). The second paper on equitable decompositions extends this to basic automorphisms in which the order of the automorphism is the product of distinct primes [10]. Most recently, the theory of equitable decompositions has been extended to any automorphism $\phi \in \operatorname{Aut}(G)$ of any order [11].

An equitable decomposition of a matrix $A$ with respect to a general automorphism $\phi$ is a more computationally expensive process than performing a FST. If the order of $\phi$ has the prime decomposition

$$
|\phi|=p_{1} p_{2} \cdots p_{\ell}
$$

where each $p_{i}$ is a prime then the equitable decomposition over $\phi$ takes $\ell$ steps; one for every prime in $|\phi|$. At each step in the process we need to create a matrix $S_{i} \in \mathbb{R}^{n \times n}$. This results in an equitable decomposition given by a sequence of similarity transforms

$$
S_{\ell}\left(\cdots\left(S_{2}\left(S_{1} A S_{1}^{-1}\right) S_{2}^{-1} \cdots\right) S_{\ell}^{-1}=A_{\phi} \oplus B \in \mathbb{R}^{n \times n}\right.
$$

Each step of the process involves a number of substeps for creating the matrix $S_{i}$ as well as creating a new automorhpism $\phi_{i}$ used in the following step to create $S_{i+1}$.

Although the process shown by Equation (6.2) results in a real decomposition of $A$, which may be useful in certain applications, a single step in this process is typically more complicated, both theoretically and computationally, than the whole of the FST method introduced in Chapter 3. Moreover, the method of equitable decompositions is, in fact, 
limited to decompositions over graph symmetries while the method presented in this paper can be used on any graphs, directed or undirected, weighted or unweighted, that have either an equitable partition, an ERP, or an ETP.

\section{Chapter 7. Conclusion}

As mentioned in Chapter 6, the FST method very much simplifies and otherwise improves the theory of equitable decompositions. It does so by extending the method of equitable decompositions that work only with graph symmetries to the more general case of equitable partitions on directed and undirected graphs.

To handle the directed case we introduce in this paper two new kinds of equitable partitions. Namely, equitable receiving partitions and equitable transmitting partitions. We show that a graph weakly decomposes under a Fourier similarity transform if and only if the graph has at least one of these partitions, and completely decomposes if and only if it has a partition that is both an ERP and an ETP simultaneously. We also show that this method preserves the eigenvalues of the original adjacency matrix, and separates them into two groups: those that are eigenvalues of the divisor graph, and those that are eigenvalues of the remainder graph.

Though the Fourier transformations decompositions may not always result in the simplest decomposition possible, e.g. the decomposition may be complex-valued while others may be real, the simplicity of the method and the if and only if relationship between the FST and graph decompositions suggest that there are more connections to be found and many of the potential applications of the FST method are currently unexplored.

For instance, how thoroughly can a FST decompose a graph? We saw in Example 3.5 that the FST decomposed into two components with real weights, Example 5.5 into two components with imaginary weights. The Paul Revere network in Figure 3.4 broke into the divisor graph and 224 isolated vertices. A potentially interesting question is whether it is possible that every graph can be decomposed as nicely, or will some graphs always have 
imaginary edges under a Fourier similarity transform? When a graph is decomposed, we are guaranteed two components (the divisor graph and some remainder) but can we guarantee any further decomposition as in Figure 3.4?

Another line of questions are related to Fourier theory and signal analysis. What would it mean for a graph to be decomposed into frequency space, which is a standard notion in Fourier theory? Are there other applications of signal processing that can be applied to graphs, such as Kalman Filtering? Could some aspect of signal processing be used to generate a useful link prediction algorithm in real-world networks, etc.? These are the kind of questions that we hope to considered in future studies on this topic. 


\section{BIBLIOGRAPHY}

[1] A. Balaban, Applications of Graph Theory in Chemistry. Journal of Chemical Information and Computer Sciences, 25:334-343, 1985

[2] W. Barret, A. Francis, and B. Webb, Equitable decompositions of graphs with symmetry. Linear Algebra and its Applications, 513:409 - 434, 2017.

[3] A. Boggess and F. J. Narcowich, A first Course in wavelets with Fourier analysis. John Wiley \& Sons, Hoboken, NJ, second edition, 2009.

[4] D. Bonchev and D.H. Rouvray (Eds.), Chemical Graph Theory: Introduction and Fundamentals. Abacus Press, 1991

[5] K.L. Calvert, M.B. Doar, and E.W. Zegura, Modeling Internet Topology. IEEE Communications Magazine, 35:160-163, 1997.

[6] P. Carrington, J. Scott, and S. Wasserman, Models and Methods in Social Network Analysis. Cambridge University Press, 2005.

[7] C. Chen, W. Ye, Y. Zuo, C. Zheng, and S. Ping Ong, Graph Networks as a Universal Machine Learning Framework for Molecules and Crystals. Chemistry of Materials, 31(9):3564-3572, 2019

[8] T. Cormen, C. Leiserson, R. Rivest, C. Stein, Introduction to Algorithms. The MIT Press, Cambridge, Massachusetts, third edition, 2009.

[9] D. Cvetkovic, P. Rowlinson, and S. Simic, An Introduction to the Theory of Graph Spectra. London Mathematical Society Student Texts. Cambridge University Press, 2009.

[10] A. Francis, D. Smith, D. Sorensen, and B. Webb, Extensions and applications for graphs with symmetries. Linear Algebra and its Applications, 532: 432 - 462, 2017 
[11] A. Francis. D. Smith, and B. Webb, General equitable decompositions of graphs with symmetries. Linear Algebra and its Applications, 577:287 - 316, 2018.

[12] G. Frederickson, Data Structures for On-Line Updating of Minimum Spanning Trees with Applications. SIAM Journal of Computing, 14(4):781-798, 1985

[13] C. Godsil and G. F. Royle, Algebraic Graph Theory. Graduate Texts in Mathematics. Springer New York, 2001.

[14] J. Gomes and L. Velho, From Fourier Analysis to Wavlets. Volume 3 of IMPA Monographs. Springer, Cham, 2015.

[15] A. Khazaee, A. Ebrahimzadeh and A. Babajani-Feremi, Identifying patients with Alzheimer's disease using resting-state fMRI and graph theory. Clinical Neurophysiology, 126(11):2132-2141, 2015.

[16] D. Koller and N. Friedman, Probabilistic Graphical Models: Principles and Techniques. The MIT Press, Cambridge, Massachusetts, 2009.

[17] A. Lerch, An Introduction to Audio Content Analysis: Applications in Signal Processing and Music Informatics. John Wiley \& Sons, Hoboken, NJ, 2012.

[18] Y. Lo, S. Rensi, W. Torng, and R. Altman, Machine learning in chemoinformatics and drug discovery. Drug Discovery Today, 23:1538-1546, 2018

[19] B. MacArthur, R. Sanchez-Garcia, and J. Anderson. Symmetry in complex networks. Discrete Applied Mathematics, 156(18):3525 - 3531, 2008.

[20] K. Murphy, Machine Learning: A Probabilistic Perspective. The MIT Press, Cambridge, Massachusetts, 2012.

[21] M. Newman, Networks: An Introduction, Oxford University Press (2010). 
[22] N. O'Clery, Y. Yuan, G. Stan, and M. Barahona, Observability and coarse graining of consensus dynamics through the external equitable partition. Physical Review E, 88(4), 2013.

[23] M. Petrou and C. Petrou, Image Processing: The Fundamentals. John Wiley \& Sons, Hoboken, NJ, 2010.

[24] T. Riedel and U. Brunner, Traffic Control using Graph Theory. Control Engineering Practice, 2(3):397-404, 1994

[25] M. Schaub, N. O’Clery, Y. Billeh, J. Delvenne, R. Lambiotte, and M. Barahona, Graph partitions and cluster synchronization in networks of oscillators. Chaos: An Interdisciplinary Journal of Nonlinear Science, 26(9), 2016.

[26] S. Shrinivas, S. Vetrivel, and N. Elango, APPLICATIONS OF GRAPH THEORY IN COMPUTER SCIENCE AN OVERVIEW. International Journal of Engineering Science and Technology, 2(9):4610-4621, 2010.

[27] A. Siddique, L. Pecora, J. Hart, F. Sorrentino, Symmetry- and input-cluster synchronization in networks. Physical Review E, 97(4), 2018.

[28] V. Wedeen, P. Hagmann, W. Tseng, T. Reese, and R. Weisskoff, Mapping complex tissue architecture with diffusion spectrum magnetic resonance imaging. Magnetic Resonance in Medicine, 54:1377-1386, 2005. 